\title{
Report of 2018 equine influenza outbreak in Chile
}

\author{
Rubén Moreira ${ }^{\mathrm{a}, b^{*}}$, Alfonso García ${ }^{\mathrm{a}}$, Carolyn Ahumadaa ${ }^{\mathrm{a}}$ Catalina Badía ${ }^{\mathrm{a}}$, Paulina Suárez ${ }^{\mathrm{a}}$, \\ Betty Yangaria $^{a}$, Carolina Aguayo ${ }^{a}$, José Herrera ${ }^{a}$, Gabriela Espejo ${ }^{a}$, Enrique Pinto ${ }^{c}$
}

\begin{abstract}
The present study reports an outbreak of equine influenza (EI) occurred in Chile in January 2018, with cases spread along the country, from the Atacama to the Magallanes Region. The virus identified corresponded to the H3N8 subtype, affecting equines, donkeys, and mules. Clinical signs ranged from mild to severe, with a higher mortality rate in donkeys.

Key words: equine influenza, outbreak, equines, donkeys, Chile.
\end{abstract}

\section{INTRODUCTION}

Since 1963 the equine influenza virus (EIV) H3N8 subtype has been the cause of numerous outbreaks of respiratory disease in horses worldwide, including South American countries where the horse industry is of major importance (Olguin Perglione et al 2016). Recognised as a common malady of equids for centuries, influenza is a highly contagious viral infection mainly characterised by fever, depression, a mucoid or mucopurulent discharge, coughing and impairment of normal behaviour (Timoney 1996). These signs usually become more evident between 1 and 2 days post-infection and can last several days (Glass et al 2002). The incubation period fluctuates depending on the preexisting immunity of the horse and the magnitude of the virus attack, but it can be as short as 24 hours (Cullinane and Newton 2013).

The EIV of the H3N8 subtype were initially assigned to a single cluster that evolved into two sublineages, American and Eurasian, according to the geographic region. The American lineage was divided into South American, Kentucky, and Florida sublineages. The strains of the Florida sublineage had amino acid mutations in the hemagglutinin (HA1) subunit, and this lineage diverged into Florida Clade1 (FC1) and Florida Clade 2 (FC2), represented by A/equine/South Africa/4/2003 and A/ equine/Richmond/1/07, respectively. The lineages FC1 and $\mathrm{FC} 2$ have been identified in outbreaks worldwide and are predominantly circulating EIVs (Favaro et al 2018).

In Chile, the first outbreak of equine influenza (EI) was described in 1963 (Fuschlocher et al 1963). In 1977, the EIV was isolated, corresponding to the H7N7 subtype (Casanova et al. 1977), later in 1985, the H3N8 subtype was isolated (Berríos et al 1986). Another outbreak was

Accepted: 12.10 .2018

aServicio Agrícola y Ganadero, Ministerio de Agricultura, Santiago, Chile.

${ }^{b}$ Facultad de Medicina Veterinaria y Recursos Naturales, Universidad Santo Tomás, Santiago, Chile.

${ }^{c}$ Facultad de Ciencias Veterinarias y Pecuarias, Universidad de Chile, Santiago, Chile.

*Corresponding author: R Moreira; ruben.moreira@sag.gob.cl. reported in Chile in March 1992 where the isolate was identified as H3N8 subtype (Celedón et al 1992). In July 2006, horses from various regions of Chile presented fever, serous nasal discharge, dry cough, anorexia, and depression. The virus was identified as EIV H3N8 subtype (Müller et al 2009). During 2012, an extensive outbreak of EI (H3N8 subtype) occurred across multiple countries in South America. The epidemic was first reported in Chile, then spread to Brazil, Uruguay, and Argentina, where both vaccinated and unvaccinated animals were affected. The equine influenza epidemic in South America in 2012 was caused by a virus belonging to Florida Clade 1, similar to those circulating in the USA in 2011 (Beuttemmüller et al 2016).

The herein presented report aims to describe the 2018 EI outbreak in Chile, and to further characterise the virus subtype and how it spread throughout the country.

\section{MATERIAL AND METHODS}

Two hundred and ninety nasopharyngeal swabs were collected between January $10^{\text {th }}$ and March $20^{\text {th }} 2018$, from horses showing typical influenza clinical signs, including nasal discharge, cough, and pyrexia. Samples were collected and processed for diagnostic testing at SAG's Lo Aguirre laboratory (Official Veterinary Service of Chile - SAG). Nasopharyngeal swabs in brain-heart infusion or UTM (Universal Transport Medium) transport mediums were processed for molecular analysis of equine influenza virus. From a $50 \mu \mathrm{l}$ aliquot of swab medium, viral RNA was extracted with the automated magnetic-bead-based MagMAX TM_96 Viral Isolation kit (Applied Biosystems, Cat. AM1836-5, Foster City, CA, USA) according to manufacturer's instructions, and using the KingFisher ${ }^{\mathrm{TM}}$ Duo Prime Purification System (Thermo Scientific). The extracted RNA was processed by real-time qRT-PCR with primers and probes to detect the matrix gene (Torchetti 2016) with a CT value of 36 (cycle threshold cut off). The primer sequences are detailed in table 1.

Two positive samples were isolated in 10-day-old embryo specific pathogen free (SPF) chicken eggs. Three to five days after isolation, allantoic fluid was collected 
and tested for haemagglutination activity (OIE 2017). All samples were put together into one batch of eggs. An aliquot of each isolate was then recovered and processed by gel-based RT-PCR to detect the hemagglutinin gene with specific primers for $\mathrm{H} 3$ and $\mathrm{H} 7$, which have amplification products of 1700 and $1800 \mathrm{bp}$ respectively (Müller et al 2005). The primer sequences are detailed in table 2 .

The obtained HA nucleotide sequences (A/equine/ Chile/2018) were edited with the BioEdit software v7.2.5. and aligned using ClustalW. The alignment used 43 reference HA sequences, including North-American, European and Argentinian isolates, as well as vaccine isolates available in Genbank. For comparative purposes, the analysis was performed with a partial HA1 sequence gene (930 bp). The phylogenetic tree was created by the maximum likelihood (ML) method using the MEGA6 software. The statistical validity of the sequence comparisons was tested by bootstrap analysis using 1,000 bootstrapped data sets.

\section{RESULTS AND DISCUSSION}

In January 2018, it was reported to SAG that pure breed Chilean horses from Colina (Metropolitan Region) were experiencing respiratory signs compatible with equine influenza virus infection. On January $11^{\text {th }}, 2018$ the affected farm was visited and samples from 3 horses were collected and submitted for analysis. The presence of EIV was confirmed by SAG Lo Aguirre's laboratory using RT-PCR technique (Muller et al 2019, Torchetti 2016). To verify the antigenic response, neuroaminidase and hemagglutinin genes were sequenced. The nucleotide sequence of hemagglutinin was submitted to GenBank (MH824795).

Table 1. Primers and probes for the detection of the matrix gene of EIV (Torchetti 2016).

\begin{tabular}{ll}
\hline \multicolumn{1}{c}{ Name } & \multicolumn{1}{c}{ 5'-3' Sequence } \\
\hline Primer M +25 & AGATGAGTCTTCTAACCGAGGTCG \\
Probe $\mathrm{M}+64$ & FAM- TCAGGCCCCCTCAAAGCCGA-TAMRA \\
Primer M-124 2002 & TGCAAAAACATCTTCAAGTCTCTG \\
Primer M-124 2009 & TGCAAAGACACTTTCCAGTCTCTG \\
\hline
\end{tabular}

Table 2. Forward and reverse primers for the detection of the hemagglutinin gene H3 and H7 subtypes. (Muller et al 2009).

\begin{aligned} & \hline Name \multicolumn{1}{c}{ 5'-3' Sequence } \\ & \hline Primer H3-F ATGAAGACAACCATTATTTTGATACTACTG \\ & Primer H3-R TCAAATGCAAATGTTGCATCTGATGTTGCC \\ & Primer H7-F ATGAACACTCAAATTCTAATATTAGCCAC \\ & Primer H7-R TCATATACAAATAGTGCACCGCATGTTTC \\ & \hline\end{aligned}

The samples 15646-24 and 15781-3 were positive in the qRT-PCR targeting the matrix gene (CT values: 19.64 and 20.42 respectively). A high percentage of similarity was obtained with virus $\mathrm{H} 3 \mathrm{~N} 8$ from different geographic areas. Specific primers for N8 were used to sequence the neuraminidase gene from the samples 15989-1 and 16186 (Killian 2013), obtaining fragments with amplification products of $1450 \mathrm{bp}$. For the interpretation of the sequence obtained, it is important to understand that since 1987, H3N8 EIV genetic evolution originated two lineages: European (Sussex 89) and American lineage (Kentucky 94). Later, American lineage originated Florida lineage, which split into two clades: Florida 1 and Florida 2 (Daly et al 2011).

The phylogenetic tree includes all lineages mentioned above (figure 1). The Genbank accession numbers of the sequences used to create the tree are detailed in table 3 . The recent Chilean isolate belongs to Clade 1, Florida lineage. North-American isolates described since 2013 belongs to this clade as well, with EIVs from outbreaks in South Africa and Australia (Daly et al 2011).

After the initial report received by the SAG, strict monitoring was performed and nasopharyngeal swab samples from suspected cases from the Atacama to the Magallanes region were collected and analysed (table 4$)$. Results showed that $242 / 290(83.4 \%)$ of the analysed samples were positive in the Real time RT PCR type A influenza matrix gene. From January $10^{\text {th }}$ to March $20^{\text {th }}$, the SAG identified 255 EI affected premises between the regions of Atacama and Magallanes, involving a total of 9,296 susceptible equidae (table 5). The presence of this disease was communicated nationwide to all SAG personnel and to the association of equine veterinary specialists. The SAG also informed private veterinarians about the outbreak to encourage EI vaccination and to stop transport of the affected animals to other sites for at least the following 21 days.

Equine influenza is a disease of mandatory reporting to SAG and international notification to the World Organisation for Animal Health (OIE). In Chile, outbreaks of the disease have occurred sporadically, for example in 2006 (Müller et al 2009) and 2012 (Beuttemmüller et al 2016) due to immune breaks caused by the discontinuation of vaccination programs. The occurrence of this endemic disease after several years could be due to poor herd immunity, caused by a decrease in the protective immunity that the vaccine confers, or it could be due to a lower application frequency of specific vaccines, particularly in the stratum equines of Chilean thoroughbred. Vaccination is not mandatory for horses; however, there are only an average of 58,000 doses available per year for immediate use. According to official data, and considering an estimated population of 300,000 equines, $20 \%$ of Chilean equines have been annually vaccinated for the past five years.

During this outbreak, the equines did not present substantial changes in the pattern of symptoms, maintaining a mild to severe condition, with cough being one of the main signs in affected horses. The disease was confirmed in a 


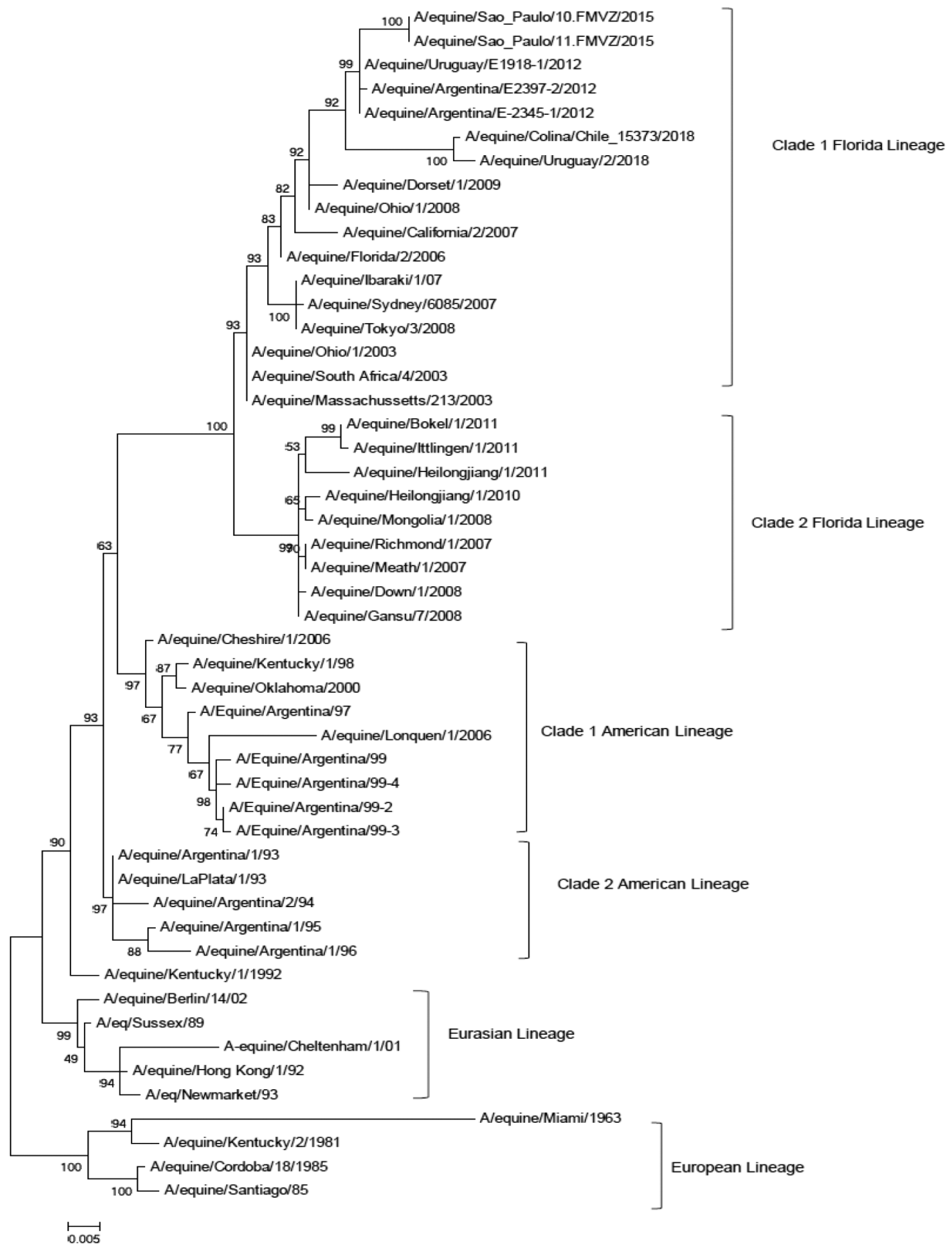

Figure 1. Phylogenetic tree created from nucleotide sequences of the HA1 portion of the haemagglutinin molecule of equine influenza H3N8 strain recently isolated in Lo Aguirre and prototype strains of the different lineages and clades. The tree was constructed using maximum likelihood (ML) method. Genbank accession numbers are available in table 3.

Thoroughbred and a Chilean pure breed horse (Caballo corralero).

In domestic and feral donkeys, the observed symptoms were respiratory distress, mucous purulent nasal secretion, and cough. Complicated cases presented mild respiratory signs, with cough, mucus, and fever. Pneumonia (pneumonic foci in the apical, iliac and ventral mediastinal regions) and death were reported in $3 / 10$ of those affected donkeys, most likely associated with secondary infection with bacterial agents, such as Streptococcus equi.

It is estimated that EIV has been circulating since December 2017 where it spread through equestrian sporting 
Table 3. Sequences used in the phylogenetic analysis and their genbank accession numbers.

\begin{tabular}{|c|c|}
\hline Name & Genbank accession $\mathrm{N}^{\circ}$ \\
\hline A/equine/Dorset/1/2009 & CY054287.1 \\
\hline A/equine/Ohio/1/2008 & GU045283.1 \\
\hline A/equine/California/2/2007 & FJ195402.1 \\
\hline A/equine/Florida/2/2006 & FJ195403.1 \\
\hline A/equine/Ibaraki/1/07 & AB360549 \\
\hline A/equine/Sydney/6085/2007 & GU045763 \\
\hline A/equine/Tokyo/3/2008 & AB435160.1 \\
\hline A/equine/South Africa/4/2003 & GU447312 \\
\hline A/equine/Ohio/1/2003 & DQ124192 \\
\hline A/equine/Massachussetts/213/2003 & DQ124193.1 \\
\hline A/equine/Heilongjiang/1/2010 & JQ265982.2 \\
\hline A/equine/Mongolia/1/2008 & AB436910.1 \\
\hline A/equine/Gansu/7/2008 & EU794495.1 \\
\hline A/equine/Bokel/1/2011 & CY100070.1 \\
\hline A/equine/Ittlingen/1/2011 & CY107012.1 \\
\hline A/equine/Heilongjiang/1/2011 & JQ265983.1 \\
\hline A/equine/Down/1/2008 & JN222937 \\
\hline A/equine/Richmond/1/2007 & FJ195395 \\
\hline A/equine/Meath/1/2007 & JN222935 \\
\hline A/equine/Cheshire/1/2006 & FJ195401.1 \\
\hline A/equine/Kentucky/1/98 & AF197241 \\
\hline A/equine/Oklahoma/2000 & AY273168.1 \\
\hline A/equine/Argentina/97 & AY048077 \\
\hline A/equine/Lonquen/1/2006 & EU926631 \\
\hline A/equine/Argentina/99 & AY048081 \\
\hline A/equine/Argentina/99-4 & AY048080 \\
\hline A/equine/Argentina/99-2 & AY048078 \\
\hline A/equine/Argentina/99-3 & AY048079 \\
\hline A/equine/Argentina/1/95 & AF197244 \\
\hline A/equine/Argentina/1/96 & AF197246 \\
\hline A/equine/Argentina/2/94 & AF197245 \\
\hline A/equine/Argentina/1/93 & L39913 \\
\hline A/equine/LaPlata/1/93 & D30686 \\
\hline A/equine/Kentucky/1/1992 & CY030149 \\
\hline A/equine/Berlin/14/02 & EF541430.1 \\
\hline A/eq/Sussex/89 & X85090.1 \\
\hline A/equine/Argentina/E-2345-1/2012 & AHJ57548 \\
\hline A/equine/Argentina/E2397-2/2012 & AOS87378 \\
\hline A/equine/Sao_Paulo/10.FMVZ/2015 & AOX49483 \\
\hline A/equine/Sao_Paulo/11.FMVZ/2015 & AOX49484 \\
\hline A/equine/Uruguay/E1918-1/2012 & AOS87385 \\
\hline A/equine/Uruguay/2/2018 & MH673717 \\
\hline A/equine/Hong Kong/1/92 & L27597 \\
\hline A/eq/Newmarket/93 & X85089 \\
\hline A/equine/Cheltenham/1/01 & EF541435.1 \\
\hline A/equine/Kentucky/2/1981 & CY028820 \\
\hline A/equine/Cordoba/18/1985 & CY032301.1 \\
\hline A/equine/Santiago/85 & AY383755.1 \\
\hline
\end{tabular}

Table 4. Nasopharyngeal swabs collected by region.

\begin{tabular}{lrrr}
\hline \multicolumn{1}{c}{ Regions } & Positive & Negative & Total \\
\hline Atacama & 3 & 0 & 3 \\
Coquimbo & 8 & 2 & 10 \\
Valparaíso & 37 & 14 & 51 \\
Metropolitana & 34 & 9 & 43 \\
O’Higgins & 9 & 2 & 11 \\
Maule & 33 & 2 & 35 \\
Biobío & 23 & 2 & 25 \\
Araucanía & 4 & 2 & 6 \\
Los Ríos & 3 & 0 & 3 \\
Los Lagos & 12 & 5 & 17 \\
Aysén & 69 & 5 & 74 \\
Magallanes & 7 & 5 & 12 \\
\hline & 242 & 48 & 290 \\
\hline
\end{tabular}

Table 5. Premises and horses affected by regions.

\begin{tabular}{lrcr}
\hline \multicolumn{1}{c}{ Regions } & Premises affected & Susceptible horses & Cases \\
\hline Atacama & 27 & 784 & 161 \\
Coquimbo & 9 & 249 & 101 \\
Valparaiso & 32 & 709 & 186 \\
Metropolitana & 28 & 4.669 & 273 \\
O’Higgins & 11 & 127 & 50 \\
Maule & 44 & 603 & 264 \\
Biobío & 34 & 843 & 170 \\
Araucanía & 12 & 139 & 62 \\
Los Ríos & 3 & 247 & 105 \\
Los Lagos & 14 & 475 & 107 \\
Aysén & 36 & 353 & 165 \\
Magallanes & 5 & 98 & 57 \\
\hline & 255 & 9,296 & 1,701 \\
\hline
\end{tabular}

events, which involved travelling of affected horses to various parts of the territory, thus explaining the rapid spread to all equids that had not been properly immunised.

The spatial pattern of the cases, indicated a distribution throughout the country from the Atacama to the Magallanes Region. The report of cases compatible with EI were notified and recorded. To characterise the real distribution and dissemination of the virus in the national equine population, samples were collected for EI molecular diagnosis. Cases were confirmed in donkeys from the Valparaíso and Atacama regions.

The north region of the country (Arica and Parinacota, Tarapacá, and Antofagasta), kept epidemiological silence and did not report suspected cases compatible with EI infection in their equine population. The index cases may not be the source of the virus, they act to amplify the virus and serve as a source of infection to other horses in the 
cohort. The severity of the disease depends primarily on the immunological condition of the horses at the time of exposure, the environment, the stress created by continuing to work or being trained (Cullinane and Newton 2013). Equine influenza is a self-limiting disease, and the virus does not persist in horses that have recovered. It is thought that influenza virus persists in endemic populations by low-grade circulation with small occasional outbreaks (Glass et al 2002).

The 2018 EI H3N8 subtype outbreak in Chile was caused by reduced vaccination and poor herd immunity of Chilean equids, particularly Pure Breed Chilean horses. Regulations that will require strategic vaccination of horses that move permanently, or temporarily for sporting purposes are being evaluated.

\section{REFERENCES}

Berríos P, Celedón MO, Sepúlveda O. 1986. Influenza equina. Aislamiento del virus (H3N8). Av Cs Vet 1, 65-66.

Beuttemmüller EA, Woodward A, Rash A, Ferraz LES, Alfieri AF, et al. 2016. Characterization of the epidemic strain of H3N8 equine influenza virus responsible for outbreaks in South America in 2012. Virol J 13, 45 .

Casanova A, Martínez I, Román M. 1977. Aislamiento y tipificación del virus de la influenza equina en Chile. Arch Med Vet 9, 91-93.

Celedón MO, De Negri L, Santibáñez M, Berríos P. 1992. Brote de influenza equina en Chile causado por el subtipo H3N8. AgroCiencia. 8, 47-48.
Cullinane A, Newton JR. 2013. Equine influenza-A global perspective. Vet Micro 167, 205-214.

Daly JM, MasRae S, Newton JR, Wattrang E, Elton DM. 2011. Equine influenza: A review of an unpredictable virus. Vet J 189, 7-14.

Favaro PF, Fernandes WR, Reischak D, Brandão PE, Silva SOS, et al 2018. Evolution of equine influenza viruses (H3N8) during a Brazilian outbreak, 2015. Braz J Microbiol. 49, 336-346.

Fuschlocher F, Zurita L, Latorre G, Palavicino I.1963. Influenza equina en la provincia de Santiago. V Convención Nacional de Medicina Veterinaria. Valdivia. Boletín. 40-46.

Glass K, Wood JL, Mumford JA, Jesset D, Grenfell BT. 2002. Modelling equine influenza 1: a stochastic model of within-yard epidemics. Epidemiol Infect 128, 491-502.

Killian M. 2013. Primer table for influenza A virus amplification and sequencing. WI-AV-0022.02. USDA-NVSL.

Müller I, Jaureguiberry B, Valenzuela P. 2005. Isolation, sequencing and phylogenetic analysis of the hemagglutinin, neuraminidase and nucleoprotein genes of the Chilean equine influenza virus subtypes H7N7 and H3N8. Biol Res 38, 55-67.

Müller I, Pinto E, Santibáñez MC, Celedón MO, Valenzuela PDT. 2009. Isolation and characterization of the equine influenza virus causing the 2006 outbreak in Chile. Vet Microbiol 137, 172-177.

OIE, Organización Mundial de Sanidad Animal, 2017. Manual of Diagnostic Tests and Vaccines for Terrestrial Animals, Chapter 2.5.7: Equine Influenza (Infection with equine influenza virus).

Perglione CO, Golemba MD, Torres C, Barrandeguy M. 2016. Molecular epidemiology and spatio-temporal dynamics of the H3N8 equine influenza virus in South America. J Pathog 5, 61-75.

Timoney PJ. 1996. Equine Influenza. Comp Immun Microbiol Infect Dis 19, 205-211.

Torchetti M. 2016. Real-time RT-PCR detection of influenza A virus and avian paramyxovirus type-1. SOP-AV-0068.02. USDA-NVSL. 
